\title{
International Chinese Students in the UK: Association between Use of Green Spaces and Lower Stress Levels
}

\author{
Song Zhao ${ }^{1, *(\mathbb{D})}$ and Agnès Patuano ${ }^{2}$ (D) \\ 1 Edinburgh School of Architecture and Landscape Architecture, The University of Edinburgh, \\ Edinburgh EH1 2LE, UK \\ 2 Landscape Architecture and Spatial Planning Group, Wageningen University and Research, P.O. Box 47, \\ 6700 AA Wageningen, The Netherlands; agnes.patuano@wur.nl \\ * Correspondence: s1809285@ed.ac.uk
}

check for updates

Citation: Zhao, S.; Patuano, A. International Chinese Students in the UK: Association between Use of Green Spaces and Lower Stress

Levels. Sustainability 2022, 14, 89. https://doi.org/10.3390/su14010089

Academic Editors: Catharine Ward Thompson and Francisca Lima

Received: 1 October 2021

Accepted: 20 December 2021

Published: 22 December 2021

Publisher's Note: MDPI stays neutral with regard to jurisdictional claims in published maps and institutional affiliations.

Copyright: (C) 2021 by the authors. Licensee MDPI, Basel, Switzerland. This article is an open access article distributed under the terms and conditions of the Creative Commons Attribution (CC BY) license (https:// creativecommons.org/licenses/by/ $4.0 /)$.

\begin{abstract}
The health benefits of urban green spaces have been found to vary for different populations, such as people of different socio-demographics or different cultures. Among these populations, one remains understudied although its numbers are growing: Chinese international students. Indeed, more and more Chinese students choose to go abroad for higher education but face specific challenges, often resulting in them experiencing high levels of stress and poor health. This study explores the link between Chinese international students' use of local green spaces and the effect on their perceived stress and health. An online survey was created to collect data from 186 Chinese international students studying in Edinburgh, Scotland (UK). The data covered the participants' reported health status and their perceived stress levels, the strategies they use when coping with stress, their awareness of the benefits of visiting green spaces as well as their self-reported use of and access to local green spaces. The findings show a significant correlation between access to and use of urban green spaces and the self-reported wellbeing indicators. Some of the barriers experienced by participants in visiting green spaces were also explored. By investigating the specific behaviors of this emerging and vulnerable population, this study expands the corpus of existing evidence for the role played by urban green spaces in supporting wellbeing. Some recommendations to support the health of this community using urban green spaces can therefore be proposed.
\end{abstract}

Keywords: urban green space; international students; landscape and wellbeing; use of green space; China

\section{Introduction}

\subsection{The Importance of Nature for Health and Wellbeing}

There is an expanding body of research studying the relationship between urban green spaces and human health [1]. Studies range from teasing out the existing associations between environmental characteristics and health status measurements [2,3] to the mechanisms underlying such relationships [4,5], along with what type of green areas or green elements are more efficient in promoting health [6-8].

Notably, some studies have focused on the impact of green space on specific populations, which might be more vulnerable to health issues, such as older people [9], ethnic minorities [10], or people in deprived communities [11-13].

This paper proposes to extend the corpus of existing evidence supporting the relationship between green spaces and health by examining an emerging population: international Chinese students in higher education. Indeed, this group represents both an ethnic and cultural minority while its members also face their own developmental challenges, making them part of another vulnerable population [14,15].

\subsubsection{The Importance of Access to Green Spaces for Coping}

Several theoretical frameworks have been proposed over the years to articulate the links between green space and health. Most notably, Roger Ulrich's psycho-physiological 
Stress Reduction Theory explains that both direct and indirect contact with nature (e.g., green space visits of views over a natural setting) can have a beneficial effect on people experiencing high levels of stress by allowing them to relax and to shift into a more positive emotional state $[16,17]$. According to Ulrich, humans are innately predisposed to find natural stimuli calming when they are non-threatening, and exposure to these stimuli leads to feelings of enhanced wellbeing and relaxation [16,17].

Similarly, the Kaplans' Attention Restoration Theory posits that natural environments are more likely to present restorative qualities which can support emotional regulation and recovery from low mood, fatigue, and stress [13,18].

Coping refers to the strategy people use to provide emotional regulation through either conscious or automatic effort to maintain or balance out their moods [18]. This process can rely on external factors such as interactions with the social and physical environment $[17,18]$. In this context, green spaces have been shown to be essential resources by providing restorative experiences such as relaxation, positive mood change, renewal of directed attention capacity, and contemplation of self [18]. Outdoor activities and exercise, natural daylight, stimulation of the senses, and aesthetic experience have also been cited as potential drivers for the health benefits of living close to natural environments [19].

Typically, visiting natural environments such as urban parks or forests has been found to support the health of populations through four pathways: physical, social, mental, and physiological [20]. These pathways are complex and not strictly distinct from each other [20]. However, generally, green spaces offer opportunities for physical activity and social contact, as well as relaxation and clean air, thereby providing a wide range of health benefits [20].

Therefore, an expanding body of studies has demonstrated that simply having access to green space could have an impact on human stress levels [11]. Previous work has shown that people living in environments with a higher percentage area of green space experienced lower stress levels than others, regardless of socio-demographic variables [11]. In this context, accessibility has been explored in various forms.

Regarding visual accessibility, following Ulrich's original findings on the view from hospital windows influencing the recovery of patients after surgery [16], the presence or not of a view of green spaces from the homes was also found to be a significant predictor of the perceived stress levels of the residents in deprived cities in Scotland [12]. Similarly, students studying in rooms with views of green landscape recovered better from stress experiences than those without [21].

Another aspect of accessibility can be understood as proximity [1]. In this case, studies have found that the distance between people's homes and the nearest green space was also correlated with the level of stress $[19,22]$. Shorter distances to green space were associated with lower self-reported stress levels, and so did having access to a private garden from home $[12,19,22,23]$.

\subsubsection{Factors Influencing the Use of Green Spaces for Health and Wellbeing}

An important mediator between the presence and accessibility of green spaces around people is whether they make use of these spaces [13]. Generally, the more frequently people visit green spaces, the less they report being affected by stress, irrespective of their genders, ages, or length of stay [19].

Furthermore, positive perceptions of the quality of local green spaces and the frequency of use of such spaces have been found to play an important role in the health benefits of urban living environments [24]. Some individual drivers of this relationship between visiting green spaces and health were also explored in previous studies. Most notably, childhood experiences of being in contact with nature have been shown to affect people's use of green space after they become adults [23]. 


\subsection{Definition of Stress and Perceived Stress}

Stress can be defined as "the process by which an individual responds psychologically, physiologically, and often with behaviours, to a situation that challenges or threatens wellbeing" [17] p. 202. Psychological responses can include emotions such as fear, anger, and sadness, whereas the physiological responses involve several internal systems, such as the cardiovascular, skeletomuscular, and neuroendocrine systems [17].

Stress responses can be triggered by both external and internal factors: life events such as moving homes or losing a loved one can be considered external stressors, but the responses they provoke are also mediated by people's individual perceptions of their stressfulness [25]. Therefore, stress is the result of the interaction between external stressors and people's reactions to those stressors [26]. As a result, it is difficult to establish an objective measure of stress such as it is experienced by the individuals.

Typically, stress can be measured through physiological data by tapping into one or several of the systems mentioned above: cardiovascular with electrocardiograms, heart rate variability, blood pressure or pulse transit time [17]; neuroendocrine with the measurement of skin conductance and cortisol [11,17,27]; and skeletomuscular through measuring muscle tension [17].

Another way to measure stress is through self-reported data [12]. A widely used method is the Perceived Stress Scale (PSS), which defines the perception of stress it measures as "the degree to which situations in one's life are appraised as stressful" [25] p. 385. Items were designed to express the degree of unpredictability, lack of control, or overload respondents experience in their lives [25]. Previous studies have found a significant correlation between PSS scores and physiological measurements of stress, which confirms the validity of this approach [11].

Stress is often regarded as one of the main threats to health and wellbeing in modern society, but it is a part of life that our ancestors also had to contend with [19]. Responses to stress were meant to increase our ability to fight or flight when confronted with a threat [19]. However, the presence of threats in our ancestors' environments was also balanced by opportunities to rest and recover [17]. By contrast, modern life is thought to expose us to another type of stress: "an imbalance between what we are able to accomplish and what is demanded of or expected from us, which can lead to a feeling of being unable to control our life" [19] p. 3.

\subsection{Chinese International Students: An Understudied Population}

On top of the stress of modern society, university students are often in a transitional phase of their lives, which comes with many pressures [26,28]. When starting at university, many students have had to leave their parental homes for the first time and confront their expectations with the reality of adult life [26]. Furthermore, students face the pressures of academic achievement and job security, as well as potential difficulties in their social lives [26]. Finally, student life can be accompanied by poor eating and sleeping habits as well as unhealthy behaviors such as smoking, drugs, or alcohol use, which can increase the student's perceived levels of stress $[26,28]$. As a result, a growing number of university students in the United Kingdom reports experiencing feelings of anxiety and psychological distress [29].

This transitional stress is compounded in the case of international students who leave their countries and cultures to study abroad $[15,30]$. Compared with local university students, they face not only 'self-imposed' stressors and 'pressures' related to achievement and self-realization [26], but also particular stressors such as 'adaption to new cultures', 'language barriers' and encountering 'a lack of understanding in a foreign university community' [14]. According to a survey on the stress level of Chinese international students living in 12 countries, including the United Kingdom, the United States, Australia, and Japan, $89.5 \%$ of the respondents reported the existence of stress [30]. In the United Kingdom, Chinese students reported being affected by a variety of stressors, including job opportunities, academic pressure, language barriers, and financial concerns [31]. There- 
fore, international students have been shown to face intercultural adaptation challenges, which are both transitional and adaptive, experiencing both culture shock, learning shock, language shock, and role shock [32].

Additionally, it is worth noting that in the context of globalization, more and more people choose to study abroad, especially Chinese students [30]. Such a phenomenon makes international students an expanding population as part of the residents living in the city. In the United Kingdom, Chinese students make up the largest cohort of international students, with 141,870 students reported to study in British universities in 2019/2020 [33]. It is also the fastest-growing population, with an increase of $56 \%$ over the five-year period 2015/16 to 2019/20 [33]. Although its numbers have been steadily growing, the population of international Chinese students, and in particular its wellbeing, remains understudied.

\subsection{Cultural and Demographic Variations in the Use of Green Spaces for Health and Wellbeing}

Generally, patterns of use of green spaces are recognized as dependent on cultural background [34] or race [35]. These differing patterns of use have been linked to the cultural influence on the self's structure and processes [36]. Indeed, people tend to display a mix of independent and inter-dependent self-constructs, placing different values on either being unique or fitting in $[34,36]$. Western cultures, as individualistic cultures, tend to primarily value independent self-constructs, whereas people from Asia or other collectivistic cultures tend to hold more of an interdependent self-concept [30,34,36]. Accordingly, motivations for outdoor activities and outdoor recreation preferences have been found to vary between the two types of self-constructs [36].

Furthermore, the use of green spaces for health and wellbeing is known to be correlated with childhood experiences [23,35] and nature connectedness [37]. However, as one of the most rapidly urbanizing countries in the world, China is recognized as not being able to provide many opportunities for childhood experiences in nature that would foster nature connectedness [38]. Therefore, people growing up in China might not be willing to visit green spaces later in their lives.

Finally, university students are in a specific age range between adolescence and adulthood. Several studies looking at teenagers' use of natural spaces have found that the population to be less connected with nature than children or adults, suggesting that adolescence might be a "time out" in people's relationship with nature [39-41]. However, the cut-off age for this "time out" has not yet been explored.

\subsection{How Chinese Students Cope with Stress at Home}

In line with much of the literature collected from a Western context, green spaces in China were found to reduce Chinese university students' uncertainty stress, with students living in cities with a higher provision of green spaces reporting lower levels of stress [42]. Viewing scenes from an urban park were also found to significantly contribute to reducing students' stress after English oral exams [27]. On campuses, open green spaces have been shown to have a positive impact on students' stress relief [43]. The health benefits of green spaces seem to vary among different types of environments, with the perceived naturalness of spaces being positively correlated with the cognitive restoration and health of students [44].

In their native country, studies have found Chinese students to have defining behavioral characteristics on university campuses [45,46]. Specifically, they tend to congregate in clusters, particularly in corner spaces and green spaces, for which they rely on landmarks such as trees or vertical elements $[45,46]$. Students were also found to heavily rely on a daily routine, often determined through the least effort routes $[45,46]$. Similarly, they appreciate the possibility of undertaking a variety of activities in a single space [46]. Although they often undertake activities as a group, they also value their privacy and places in which they can be alone $[45,46]$. 


\subsection{Research Questions and Aims}

There is currently a growing number of studies focused on university campus green spaces, covering students' perceptions and use of such spaces $[35,47]$ and their impact on students' perceived stress levels [35] or self-reported quality of life [48]. However, the use of green space by Chinese international students studying abroad and its potential health benefits have still not been explored.

The originality of this study, therefore, lies in its investigation of whether the wellknown links between urban green spaces and health [1] are also relevant to a vulnerable minority [14] which is currently growing in cities with universities [30] and which is likely to have come from an urban context in a rapidly urbanizing country with different cultural patterns of green space use [36,44] and stress management [15]. Based on the combined evidence on the role of childhood experiences and nature connectedness in the use of green spaces for health $[23,35,37,38]$, it can be hypothesized that Chinese university students might not visit green spaces often and might not perceive many benefits from these visits. Considering the differing patterns of use for various cultures [34-36], it can also be assumed that international Chinese students exhibit specific behaviors in their use of green space as a way to cope with stress. As they are shown to experience high levels of stress [14,15,30,32], the potential for local green spaces to support the health of international students still needs further exploration.

Therefore, this study focuses on how urban green spaces are used by Chinese international students in their host country and whether they play a role in their stress management. Our main research question is: What is the association between access to green space and the perceived stress/wellbeing of Chinese international students in the UK?

In order to answer this question, the study also considers which factors influence the use of urban green space by Chinese international students and the coping strategies they are most likely to use when faced with stress.

\section{Materials and Methods}

\subsection{Study Design}

The study was conducted in the city of Edinburgh, Scotland, United Kingdom, with the international Chinese students studying at the University of Edinburgh as the target population.

This study was based on a cross-sectional design, and data was collected in the form of an online survey. Mixed methods, both quantitative and qualitative, were applied in the survey for a more comprehensive understanding. Information on participants' contact with urban green spaces, their self-reported health status, and coping strategies was also collected (see Appendix A-Questionnaire).

In this study, contact with green space was considered from two perspectives: selfreported use of and perceived access to green space. The self-reported use of green space was estimated through multiple variables, including frequency of visits and time spent in local green spaces. These variables have been applied in a number of studies on people's use of green spaces $[12,19,49]$. Perceived accessibility was estimated as the perceived travel time to the nearest green space, the presence of a view on greenery, and of a garden around the home. View of greenery has been widely used in previous studies examining the relationship between the accessibility of green spaces and human wellbeing in a range of contexts, such as workplaces [49], deprived urban communities [12], and high school landscapes [21]. The presence/absence of a garden around the home was another widely used measurement, referenced as 'a private garden or balcony' [12]. Perceived travel time to the nearest green space was previously found to be an important factor in relation to stress and use of local green spaces $[19,22,50]$. It has also been compared with objective measures of distance and found to be a suitable surrogate for accessibility and, in some cases, a better predictor for health outcomes [22,50]. Wellbeing was assessed through participants' self-reported general health and perceived stress levels using the PSS scale [11,25]. 
Demographic data such as gender, age, and length of stay in the host country were investigated for their role in mitigating associations between access to green spaces and health benefits. Additionally, in order to explore cultural practices, participants were asked to rank their preferred coping strategies to deal with stress. A similar question on participants' favorite way of coping with stress has been asked in previous research: "What would you recommend a close friend to do if he or she felt stressed and worried?", exploring the relationship between green space measures and stress levels [19]. The participants were questioned about their belief regarding the potential of visiting green spaces as a way to cope with stress. Similar questions were asked in a study interviewing Chinese people living in Austria about their awareness of the benefits of being in natural areas [51] in order to get a full picture of the participants' perception of local green spaces.

\subsection{Study Setting}

\subsubsection{Urban Green Space Setting}

Edinburgh was reported to be the top city for green space in 2017 based on the satellite maps, with $49 \%$ of green space [52]. Figure 1 is based on the map of different types of green spaces in Edinburgh [53] and shows the many public urban green spaces accessible to students.

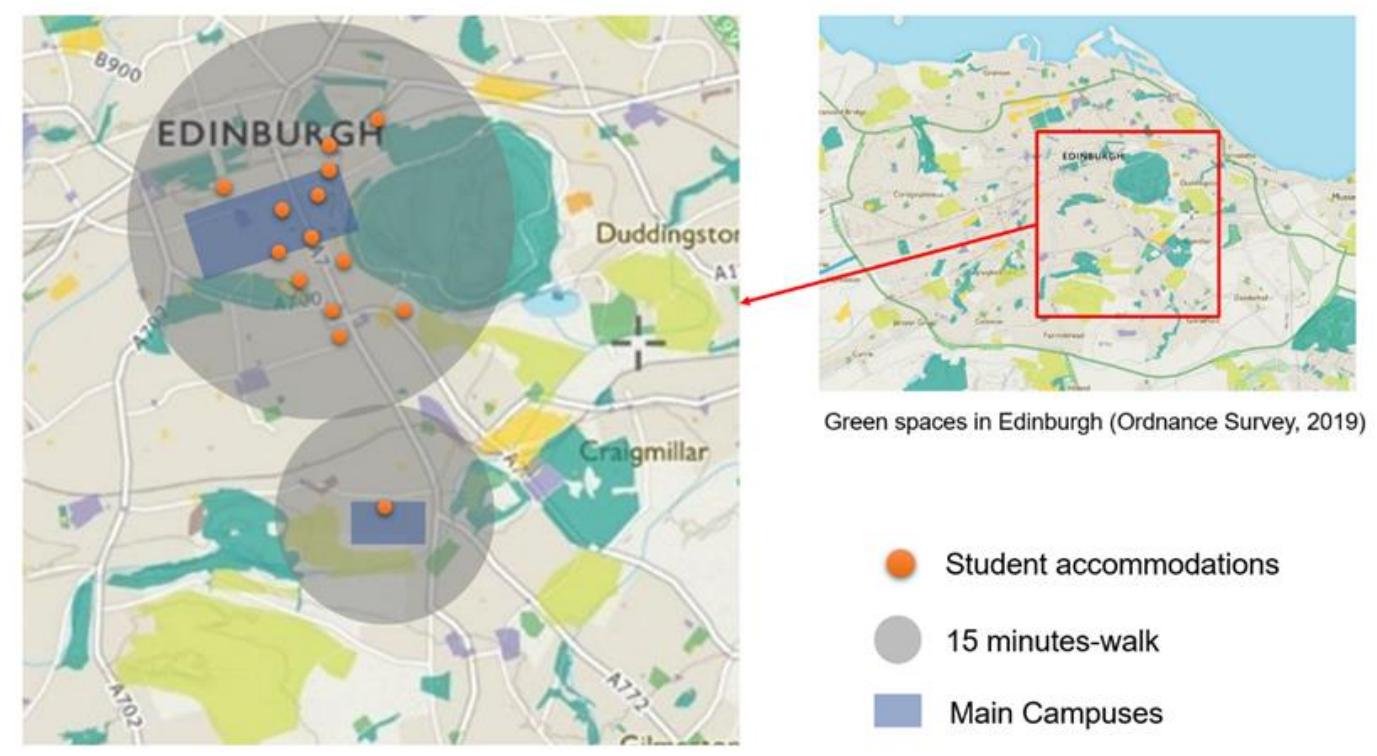

Figure 1. Map of the studied area showing students' accommodations, study spaces, and accessible green spaces.

The green space measure was based on the definition proposed by WHO [1], itself based on the definition from the European Urban Atlas, which includes parks, sports fields, natural environments such as woodlands, wetlands, and other types of ecosystems. The studied scope of green space was presented in the questionnaire. Additionally, examples of common urban green spaces in Edinburgh were provided in the instructions so that respondents could understand exactly what was meant. In this paper, "green space" refers to public urban green spaces, and the term "garden" refers to the participants' private gardens.

As presented in Figure 1, the main campuses of the university and the student accommodations are located at many different nodes in the city center, offering the students accessibility to a wide variety of urban green spaces within a 15 minute walk in their daily routines. 


\subsubsection{Student Population Setting}

The study was conducted in July 2019, with international Chinese students studying at the University of Edinburgh (UoE) as the target population. From 2017 to 2018, 3642 Chinese students (undergraduate and postgraduate) were reported to be studying in UoE, which accounted for $27 \%$ of all registered international students [54].

\subsection{Data Collection}

Participants were recruited from three Wechat chatting groups, founded and managed by the Edinburgh University Chinese Students Scholars Association (EUCSSA). Each of the groups contained 360 to 430 Chinese students studying in UoE, and the identity of each member in the groups was confirmed by the administrator as students enrolled in the 2018/19 academic year.

Due to the lack of previous literature on the topic, a focus group with students $(\mathrm{N}=13)$ from the target population was conducted in order to define potential questions within the questionnaire. The participants were volunteers in the Wechat groups. The group was of mixed gender (38\% male, $62 \%$ female) and mixed length of stay in Edinburgh (ranging between 8 months to 15 months). The results demonstrated scales of options to the questions covering the frequency of visits to green spaces, perceived travel time to the nearest green space, and time spent in such spaces per week.

The refined questionnaire was then posted by advertisements explaining the purpose of the study and instructions in the groups between 10 and 18 July 2019. Anyone who was willing to participate in the survey could click on the link and complete the questionnaire. Although every effort was made to obtain a representative sample of the general population of Chinese students matriculated at the University of Edinburgh in 2018/2019, no complete data on the student number or gender ratio could be found.

This research had gone through the Level 2 ethical approval by Edinburgh College of Art, UoE. The written consent of the participants was also collected before the start of the investigation.

\subsection{Measures}

The main content and structure of the questionnaire (translated from Mandarin) are shown in Appendix A-Questionnaire.

\subsubsection{Health Indicators}

The perceived general health was measured through a single-item question asking the participants to assess their health status in general, ranked on a 5-step scale from 1 (very poor health) to 5 (very good health). Such methodology was proposed by [55] and applied by many studies on the relationship between green spaces and human health $[5,56,57]$.

The perceived stress level (PLS) was measured using the Perceived Stress Scale (PSS) [25], which has been widely used as a reliable instrument assessing stress levels in the field of landscape and wellbeing [11]. The PSS consists of 10 items asking participants about their feelings and thoughts during the last month and to rate their frequency on a 5-point scale from 0 (never) to 4 (very often). The calculated results represent the perceived level of stress, with a higher score representing a higher stress level.

Participants were asked to write down their preferred coping strategies to deal with stress in an open question. Examples such as 'listening to music', 'take a walk' and 'exercise' were provided.

\subsubsection{Contact with Green Spaces}

In this study, contact with green space was considered from two perspectives: selfreported use of and perceived access to green space, as well as the motivations and barriers for visiting such spaces. 
1. Awareness: participants were questioned about their awareness of the health benefits of green spaces by asking whether they regarded visiting green spaces as a way of relieving stress.

2. Self-reported use of green space: frequency of visits and time spent in green spaces per week were collected with 5 options identified from the pilot work. The respondents who reported never using such spaces were asked about the reason for not visiting in an open question. The others were questioned about their motivations for visiting, activities undertaken, and the names of locations they usually visited in open questions.

3. Perceived accessibility: the perceived travel time to the nearest green space, the presence of a view on greenery and of a garden around the home.

\subsection{Main Analysis}

The qualitative information collected (stress coping strategies, reasons for not using local green space, activities undertaken in green space, and names of frequently visited green space) was analyzed using NVivo 11. Structural coding, as a widely used approach to coding [58], was performed for thematical analysis of the responses. Themes and categories were created and sorted by proportion.

Scores of the PSS were aggregated and imported into SPSS along with other collected quantitative data. Frequency and descriptive analysis were run first to get a full picture of the characteristics of participants' health status, use of, and access to local green spaces. As the data did not meet Kolmogorov-Smirnov criteria for normality of distribution, several non-parametric correlation tests, such as Spearman's non-parametric correlation test and Kruskal-Wallis test, were conducted to detect potential correlations between health indicators and measures of green space use. The correlated variables were used to build regression models predicting health or lower stress levels to gain a deeper understanding of the associations.

Effect modification by individual factors (gender, age, length of stay) was explored in two ways: (i) Mann-Whiney U Test and Kruskal-Wallis Test between individual factors, green space measures, and health indicators; (ii) regression analysis for further detection. The regression models were built using the "enter" method, so all independent variables likely to predict health or stress were entered in a single block, with the model only considering the ones significantly related to the dependent variable. This allowed the consideration of all the variables in the order of their effect on health and stress levels.

\section{Results}

\subsection{Characteristics of Participants}

The sample consisted of 186 students, among which 66 were male (35\%), and 120 were female $(65 \%)$. The majority of participants were aged between 20 and 25 years old $(n=152$, $82 \%)$. In addition, 140 (75\%) have been living in Edinburgh for less than a year.

Scores of self-reported stress levels ranged from a minimum of 7 (low stress) to a maximum of 31 (high stress), with a mean value of 18 ( $\mathrm{SD}=5.65$, moderate stress). Perceived general health ranged from very poor to very good with a median of 3 . None of the variables was found to be normally distributed; therefore, non-parametric tests were used for the rest of the analysis.

\subsection{Coping Strategies}

Figure 2 shows the coping strategies provided by the respondents. Responses were summarized and categorized by themes. 'Help and social support seeking', and 'direct action' were problem-focused coping strategies, describing the way students faced the origins of their stress and tried to provide solutions to address their problems. Students who gave answers in these categories would seek help by, for example, talking with their friends or families about the pressures they faced or dealing with the problems that caused their stress directly. Participants specifically mentioned actions like 'Seeking help from my friends/parents'. 


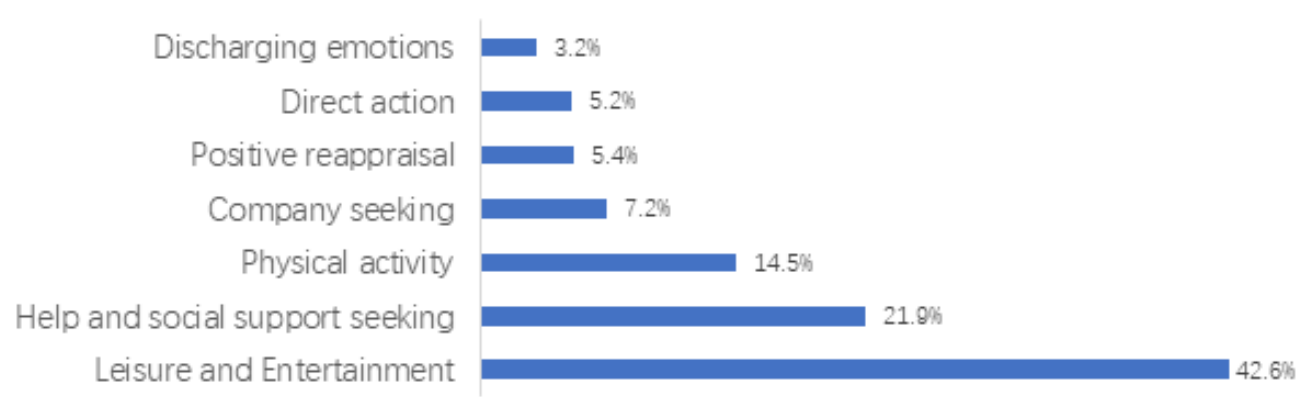

Figure 2. Summary of reported coping strategies, organized by the percentage of respondents citing them.

The emotion-focused coping strategies consist of behaviors used to relieve the discomfort caused by stressors. 'Leisure and entertainment', which was the most cited coping strategy, comprised recreational activities such "Listening to music", "watching movies" and "playing video games".

'Physical activity', including jogging, ball games, going to the gym, etc., was another important category of coping strategies. 'Company seeking' summarized the situations when participants would like to stay with someone else for relieving stress, like "hanging out with friends" and "having phone calls with friends/parents in China". Positive reappraisal contained strategies such as "thinking" and "calming down and trying to solve the problem faced". Discharging emotions like "having a hard cry" and "getting drunk" was the least cited emotional strategies.

\subsection{Use of Green Spaces and Students' Daily Life}

The majority of participants (51\%) reported visiting green spaces at least once a week and spending less than an hour there on average (Figure 3).

\section{FREQUENCY OF VISITING LOCAL GREEN SPACES}

anever

- 4 to 5 times per week

- Once a week

$=2$ to 3 times per week

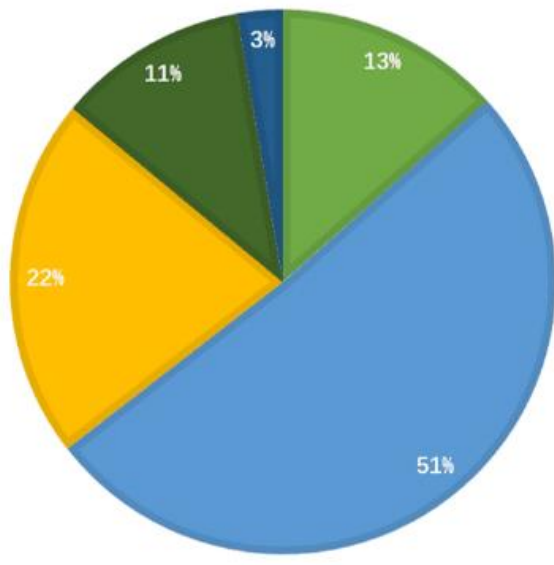

\section{TIME SPENT IN LOCAL GREEN SPACES EACH TIME}
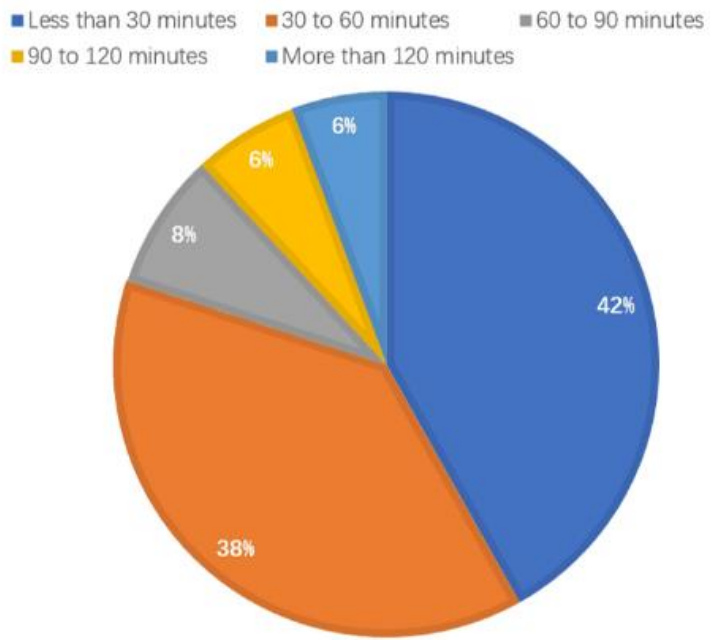

Figure 3. Descriptions of students' use of green spaces.

Additionally, a list of activities students usually preferred to undertake in green areas is presented in Figure 4. Eight categories were summarized and classified. Notably, 'taking a walk' was an important activity, taking almost $40 \%$ of all responses. 'Relaxation' mainly included students using the green spaces around the university and accommodations to relax and relieve stress in their spare time; "being in contact with nature" consisted of watching wildlife and enjoying the scenery; "picnic activities", primarily BBQ, was also widely conducted; "sunbathing" and "social interaction", such as meeting with friends, 
played essential parts in green activities; "passing through" was a kind of activity that students who lived close to green spaces often carried out on their ways; "physical exercise" including soccer, tennis, mountain climbing, and jogging was the least common green activity.

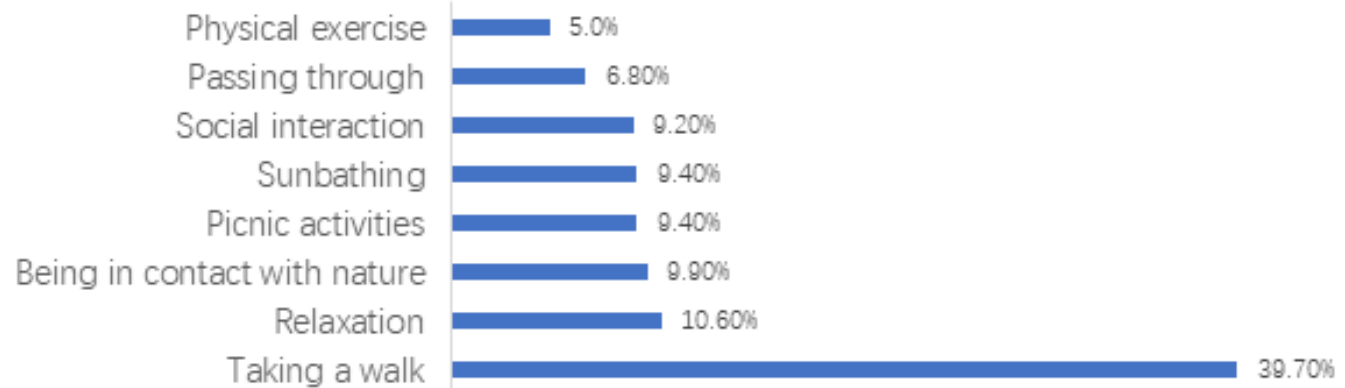

Figure 4. Summary of reported activities undertaken in green spaces, organized by the percentage of respondents citing them.

Figure 5 displays the locations of the green spaces frequently visited by the participants along with the important nodes related to students' daily routines. It illustrates that most of the frequently used green spaces submitted by students are close to their accommodations and campus, especially within a 15 min-walk.

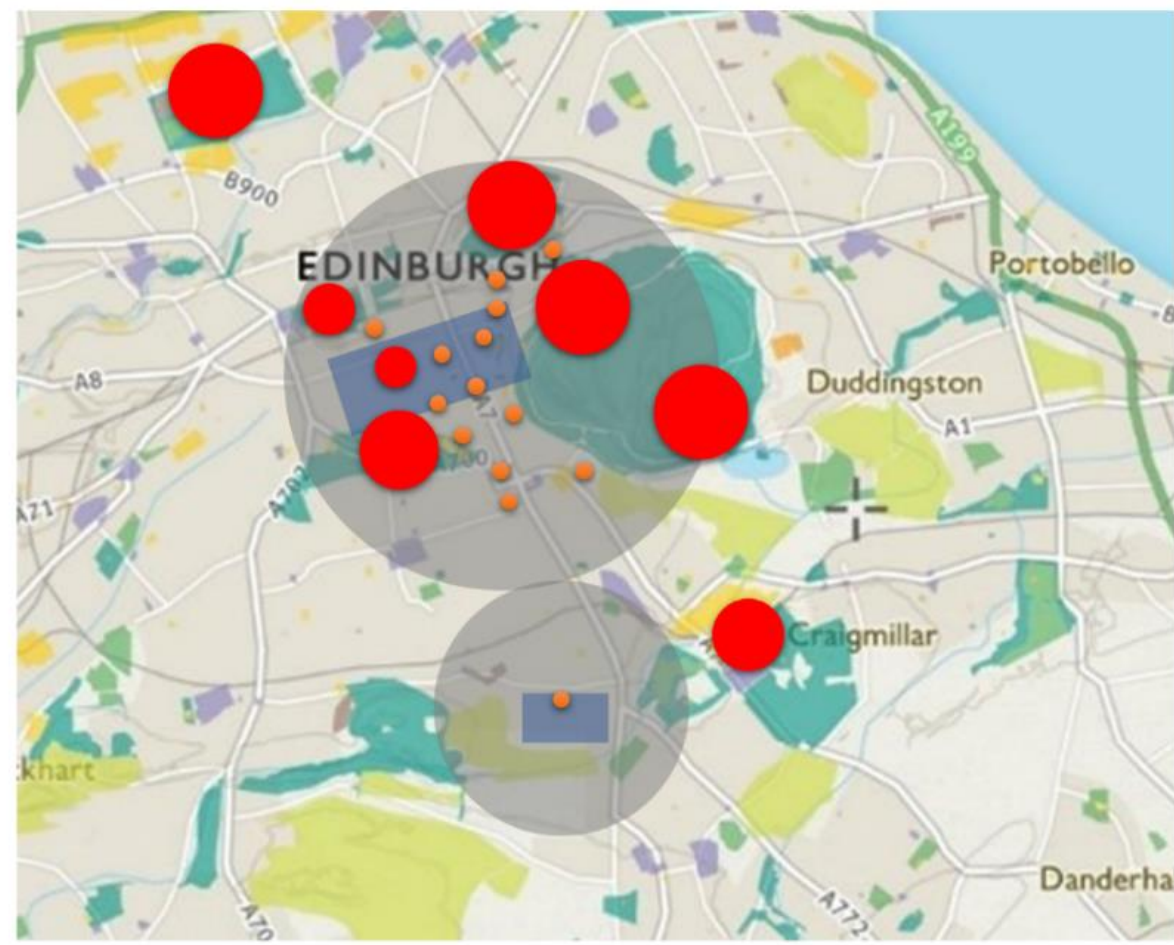

Student accommodation

15 minutes-walk

Main Campuses

Frequently visited green spaces submitted by the participants

Figure 5. The locations of frequently visited green spaces submitted by the participants.

Respondents generally had a positive opinion of the quality of green spaces in Edinburgh. Green spaces were given an average score of $4.37 / 5$, and $90 \%$ of respondents rated them as Good or Very Good.

\subsection{Relationships between Variables Related to the Use and Access to Green Spaces}

Using Spearman's non-parametric correlation test, different correlations between variables could be observed. 
As expected, the frequency of visits to green spaces was negatively correlated with perceived distance $\left(R_{S}=-0.439, p<0.0001\right)$. It was positively correlated with the time spent in green spaces, indicating that participants who visit green spaces more often also spend more time there $\left(R_{S}=0.489, p<0.0001\right)$.

An unexpected result was found regarding the attitudes of participants, with a significant correlation observed between the awareness of the benefits of green space and the self-reported use of green spaces: frequency of visit: $R_{s}=0.196, p=0.007$; time spent during visit: $R=0.236, p=0.003$. No other significant correlation could be found between the variables. To check the differences between populations in the survey, a Mann-Whitney $\mathrm{U}$ test was run using an aggregated scale variable representing the use of green spaces (frequency + time). Although neither gender nor access to a garden was related to the use of green spaces, the view of green spaces from home seemed to have an effect. Participants who had a view over green spaces from their homes were found to significantly use green spaces more $(\mathrm{U}=3836, p=0.013)$.

Tables 1 and 2 present regression models to predict the use of green spaces based on the collected variables. Awareness and perceived travel time were found to be significant predictors of the frequency of visits, as well as time spent in green spaces per week, confirming that the closer the students live to green spaces, the more they will use such spaces. No statistical relationship between perceived quality and use of green space was detected.

Table 1. Regression model predicting the frequency of visits in green spaces $(\mathrm{N}=186)$.

\begin{tabular}{|c|c|c|c|c|c|c|}
\hline & \multicolumn{2}{|c|}{ Unstandardized Coefficients } & \multirow{2}{*}{$\begin{array}{c}\text { Standardized } \\
\text { Coefficients Beta }\end{array}$} & \multirow{2}{*}{$\mathbf{t}$} & \multirow{2}{*}{$p$} & \multirow{2}{*}{$\mathbf{F}$} \\
\hline & B & Std. Error & & & & \\
\hline (Constant) & 2.313 & 0.369 & & 6.264 & $0.000^{* *}$ & \\
\hline Awareness & 0.239 & 0.082 & 0.191 & 2.924 & $0.004 * *$ & $25.821\left(0.000^{* * *}\right)$ \\
\hline Travel Time & -0.437 & 0.068 & -0.423 & -6.478 & $0.000 * *$ & \\
\hline
\end{tabular}

Dependent variable: frequency of visits. ${ }^{* *}$ Significant at the $p<0.01$ level (two-tailed). Note: the model was significant at $p<0.01$ with an $\mathrm{R} 2$ value of 0.220 .

Table 2. Regression model predicting time spent in green spaces $(\mathrm{N}=186)$.

\begin{tabular}{ccccccc}
\hline & \multicolumn{2}{c}{ Unstandardized Coefficients } & $\begin{array}{c}\text { Standardized } \\
\text { Coefficients Beta }\end{array}$ & t & $p$ & F \\
\cline { 2 - 3 } & B & Std. Error & & & 1.196 & 0.233 \\
(Constant) & 0.614 & 0.514 & 0.235 & 3.642 & $0.000^{* *}$ & $12.009\left(0.001^{* *}\right)$ \\
Awareness & 0.414 & 0.114 & -0.221 & -3.173 & $0.002^{* *}$ \\
Travel Time & -0.298 & 0.094 & & \\
\hline
\end{tabular}

Dependent variable: time spent in green spaces. ${ }^{* *}$ Significant at the $p<0.01$ level (two-tailed). Note: the model was significant at $p<0.01$ with an $\mathrm{R} 2$ value of 0.116 .

\subsection{Use of Urban Green Spaces and Wellbeing}

Using the same non-parametric correlation test, perceived general health was significantly correlated to the use of green spaces: frequency of visit: $R_{S}=0.504, p<0.000$; time spent during visit: $R_{S}=0.436, p<0.000$. It was also negatively correlated with perceived travel time to green spaces $\left(R_{S}=-0.318, p<0.000\right)$, but this might be explained by the existing correlations between the variables related to access and use of green spaces. Similarly, awareness of the benefits of green spaces were also correlated with positive general health $\left(R_{S}=0.190, p=0.009\right)$ and negatively correlated with perceived stress $\left(R_{S}=-0.181\right.$, $p=0.013)$. Perceived stress was also negatively correlated with the use of green spaces: frequency of visit: $R_{S}=-0.555, p<0.000$; time spent during visit: $R_{s}=-0.597, p<0.000$.

As expected, the two health variables, perceived general health, and perceived stress, were also correlated to each other: $R_{S}=-0.475, p=0.000$, confirming that participants who suffer more from stress also feel in worse health. 


\subsubsection{Association with Self-Reported Health}

To test the association between students' use of green spaces and their perceived general health for potential confounding variables, a linear regression was run to predict perceived general health, with gender, age, length of stay in Edinburgh, frequency of visits, and time spent in green spaces per week as the independent variables. The independent variables were entered into the model using the 'enter' method. However, only two variables, frequency and time spent, were significant. The model showed that the use of green spaces were the significant predictors of the perceived general health status (see Table 3).

Table 3. Regression model predicting perceived general health.

\begin{tabular}{|c|c|c|c|c|c|c|}
\hline & \multicolumn{2}{|c|}{ Unstandardized Coefficients } & \multirow{2}{*}{$\begin{array}{c}\text { Standardized } \\
\text { Coefficients Beta }\end{array}$} & \multirow{2}{*}{$\mathbf{t}$} & \multirow{2}{*}{$p$} & \multirow{2}{*}{$\mathbf{F}$} \\
\hline & B & Std. Error & & & & \\
\hline (Constant) & 2.098 & 0.159 & & 13.221 & $0.000 * *$ & \\
\hline Frequency & 0.294 & 0.086 & 0.304 & 3.411 & $0.001^{* *}$ & $37.909(0.000 * *)$ \\
\hline Time spent & 0.208 & 0.066 & 0.281 & 3.153 & $0.002 * *$ & \\
\hline
\end{tabular}

Dependent variable: perceived general health. ${ }^{* *}$ Significant at the $p<0.01$ level (two-tailed). Note: the model was significant at $p<0.01$ with an $\mathrm{R} 2$ value of 0.293 .

\subsubsection{Association with Perceived Stress}

Similarly, to test the association between the use of green space and stress level for potential influencing variables, another linear regression model was built to predict the levels of stress. As presented in Table 4, frequency and time spent were significant predictors for perceived stress levels. Perceived stress levels are negatively associated with the use of green spaces, showing that the more frequently students visit green areas and the more time spent in these places, the less they report feeling stressed.

Table 4. Regression model predicting perceived stress level (PSS).

\begin{tabular}{|c|c|c|c|c|c|c|}
\hline & \multicolumn{2}{|c|}{ Unstandardized Coefficients } & \multirow{2}{*}{$\begin{array}{c}\text { Standardized } \\
\text { Coefficients Beta }\end{array}$} & \multirow{2}{*}{$\mathbf{t}$} & \multirow{2}{*}{$p$} & \multirow{2}{*}{$\mathbf{F}$} \\
\hline & B & Std. Error & & & & \\
\hline (Constant) & 24.927 & 0.871 & & 28.624 & $0.000^{* *}$ & \\
\hline Frequency & -1.050 & 0.473 & -0.176 & -2.220 & $0.028 *$ & $71.247\left(0.000^{* *}\right)$ \\
\hline Time spent & -2.389 & 0.362 & -0.524 & -6.601 & $0.000 * *$ & \\
\hline
\end{tabular}

Dependent variable: perceived stress level. ${ }^{*}$ Significant at the $p<0.05$ level (two-tailed). ${ }^{* *}$ Significant at the $p<0.01$ level (two-tailed). Note: the model was significant at $p<0.01$ with an R2 value of 0.438 .

\subsection{Accessibility of Urban Green Spaces and Wellbeing}

As the accessibility of green spaces, and in particular, the perceived travel time from the home and visual accessibility through the view of green spaces from home were shown to be correlated with use, it could be established that accessible green spaces are also correlated with better wellbeing. Mann-Whitney and Kruskal-Wallis tests confirmed the association between perceived access to green spaces and perceived health and stress. Again, no significant association was detected for gender, age, and length of stay.

However, participants who never visited green spaces but had a view of greenery from home were also found to also report lower stress levels $(U=36, p=0.022)$, indicating an association independent of use. This was not the case regarding general health $(\mathrm{U}=83$, $p=0.769$ ).

Although the presence or absence of a garden was found not to be correlated with the use of green space, participants who did not visit green spaces but who did have a garden around their homes reported significantly lower stress levels $(U=15, p=0.001)$., and better perceived general health $(\mathrm{U}=28, p=0.012)$. 


\subsection{Attitudes towards Urban Green Spaces}

Seventy-seven percent of the participants agreed with the statement that visiting green spaces was a way to relieve stress. Although an awareness of the health benefits of green spaces was found to be correlated with both use of green spaces and health variables, participants were also asked about their reasons for not visiting local green spaces.

As shown in Figure 6, the reasons submitted by the participants who reported never using green spaces in Edinburgh in the past four weeks were categorized into five different themes. 'Lifestyle' and 'uninterested in visits' pointed to personal ways of living. 'Distance' and 'lack of time' confirmed the importance of the accessibility of green spaces.

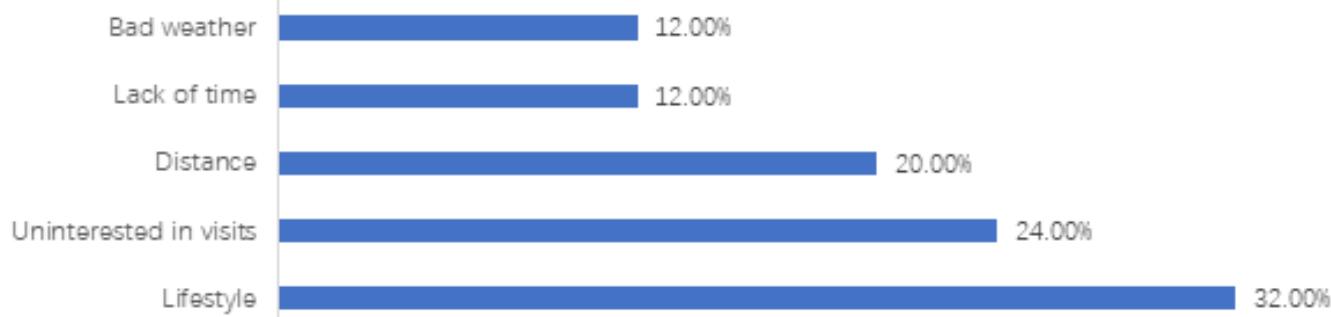

Figure 6. Summary of reported reasons for not visiting local green space.

Surprisingly, even among participants who reported never visiting green spaces, those who showed an awareness of the benefits of green spaces were still found to have better perceived general health $\left(R_{S}=0.410, p=0.042\right)$, although this effect could not be observed with perceived stress.

\section{Discussion}

\subsection{Use of Urban Green Spaces}

\subsubsection{Health Outcomes}

Consistent with previous findings, participants' reported use of green space (frequency of visits and time spent in green spaces) showed a significant association with their self-reported general health and perceived stress. Moreover, no significant differences were found regarding the health benefits of green spaces that could be linked to gender, age, or length of stay of the individuals, which was in line with previous experimental evidence [19].

Our results (Figure 2) also show that participants preferred coping with stress through seeking social support and engaging in physical activities. Accordingly, it was also found that when visiting local green spaces, students enjoyed taking a walk, going for picnics, doing exercises, and meeting with friends (Figure 4). Therefore, it appears the local green spaces provide spaces for Chinese international students to perform social and physical activities, which allows them to cope with stress. This is in line with previous findings, where physical activities [59] and social interactions [2,12] were articulated as the possible mediators of the associations between the use of green spaces and stress reduction and health promotion.

\subsubsection{Predictors of the Use of Green Space}

If the importance of the use of green spaces for health promotion and stress reduction in Chinese students can now be established, more evidence on the predictors improving the use of local green spaces by this population is needed. By testing the associations between variables, it was found that the perceived accessibility of green spaces had an impact on students' use of these spaces.

Firstly, it was detected that the perceived travel time to the nearest green space was a significant predictor of the frequency of visits. In other words, the fewer times students need to walk to green spaces, the more frequently they will visit them, supporting the 
existing literature on the importance of the proximity of green spaces for the active use of such spaces $[19,22,23,43]$.

Secondly, a view of green spaces from the participants' homes also showed a positive correlation with their use of green space, referring to both frequencies of visits and the time spent there, providing some evidence that having visual accessibility of nature from home may also lead one to spend more time in green areas. Such a finding could support the assumption made in a previous study [19] that having a view over green areas immediately from one's home may also tempt one to spend time in other green spaces.

\subsubsection{Barriers}

In our sample, the majority of participants agreed that visiting green spaces was a way to relieve stress. However, some students still did not make use of these spaces. It is, therefore, interesting to consider which barriers these participants faced.

The results showed that lifestyle and not being interested in visits were the most important reasons (Figure 6). This could be seen as consistent with the findings of [23] and the role played by childhood experiences in mitigating the use of green spaces later in life. It could indeed be assumed that some students are not in the habit of visiting such spaces, which might be the result of a variety of factors such as culture or previous living environments. Indeed, the use of green spaces for health and wellbeing is also linked to nature connectedness [37] and familiarity [41]. As China is undergoing rapid urbanization to the detriment of nature connectedness and contact with nature [38], Chinese students might now be more familiar with urban areas and feel less inclined to visit green spaces.

Moreover, distance and lack of time were the other factors cited, confirming previous findings on proximity and previous studies on the use of green spaces from Chinese students in their native country, where students were found to prefer visiting the facilities available through the least effort routes $[44,45]$. However, more research is needed to explore the specificities of this population.

Finally, Scotland has a temperate oceanic climate which can be rather cold in winter. Consequently, the weather was also cited as a barrier prohibiting students from visiting green spaces. Comparatively, the climate of China is very varied, but much of the population lives in a subtropical climate, which is warmer and with longer summers than in Scotland, which might exacerbate this barrier for the participants.

\subsubsection{Comparison with Other Populations}

Studies conducted in the UK and America both showed a significant association between university students' general health, perceived stress levels, self-estimated quality of life, and frequent use of green spaces, especially for active use of such spaces [35,48], which is in line with our results. Similarly, university students in the UK were found to use green spaces primarily to relax and socialize [47], which was consistent with our findings.

One of the main differences found in our survey, however, is regarding the coping strategies used by Chinese international students. Indeed, a study on deprived urban neighborhoods in the UK found that youths (16-25) were more likely to cope with stress by seeking company or going for a walk [13]. By contrast, in our sample, participants primarily cited using Leisure and Entertainment as a coping strategy, indicating they were more likely to use escapism to distract themselves from stressors.

Furthermore, British university students were reported to primarily use green spaces located on campuses and adjacent to the most used buildings [47]. American students in the US cited 'not being aware of opportunities' as one of the most important barriers to using green spaces [35]. By contrast, in our sample, participants were found to not use green spaces for lack of interest, regardless of whether they were aware of the many available opportunities. 


\subsection{Accessibility of Green Spaces and Health Outcomes}

Besides the finding that students who had a view of greenery from home or lived closer to green space would also use local green spaces more, there seems to be an independent association between visual accessibility and health. Indeed, within the group of students who reported never visiting local green spaces, those who had visual accessibility to green spaces reported being less stressed than those without. Supporting this assumption, previous studies have shown that viewing nature through a window or even simply watching pictures of nature can have a positive effect on people's health and wellbeing $[12,16]$, so the view of greenery from their homes might also have direct effects on students' health promotion.

Regarding access to a private garden, although previous studies showed a significant association with stress levels $[12,19,22]$, our results demonstrated inconsistent conclusions. Indeed, no such associations were detected in the main population of the sample. However, within the group of students who reported never visiting local green spaces, those who had a garden around their accommodations also reported better perceived general health and lower perceived stress levels than those without. Such findings might stress the importance of green spaces close to students' daily routines again.

\subsection{Attitudes and Coping Strategies}

\subsubsection{Opinion of Green Space Quality}

Although previous studies found a link between the perceived quality and the use of green spaces [3,24], our results show no significant association. This could be explained by the relatively homogeneously high quality of the green spaces as assessed by the students.

\subsubsection{Awareness of Health Benefits of Green Spaces}

An interesting finding of the survey was that most students who took part in the survey agreed that visiting green spaces was a way to relieve stress.

This was an unexpected result, as the variable was originally included in the survey to tease out the importance of green spaces in Chinese culture. However, similar results have been found in Austria in a study evaluating the outdoor recreation habits of two immigrant communities (Turkish and Chinese) [51]. Indeed, participants from the Chinese community were found to show great awareness of the benefits of being in natural areas and expressed the importance of being in nature [51].

Additionally, this variable was found to be one of the main factors influencing the use of green spaces and to even be correlated with perceived health for students who did not visit green spaces.

The observed correlation between this awareness and the perceived health status is particularly interesting, although it does not extend to perceived stress levels. This result could potentially be explained by the importance of internal factors influencing the perception or the experience of stress. It is possible that simply knowing that they could visit green spaces to cope with their emotions should they need to is enough for Chinese international students to improve their perceived health. However, more evidence is needed to understand the link between this awareness and the perceived health of populations.

\subsubsection{Potential Coping Strategies in Relation to Green Spaces}

From the data collected, and although participants showed overt awareness of the health benefits of visiting green spaces, coping strategies directly related to green spaces were rarely mentioned by the respondents (see Figure 2).

Instead, many of the participants (23\%) reported coping with stress by seeking help from their friends/parents. This finding was consistent with a previous study conducted in the UK [30], showing that international Chinese students preferred to endure problems when feeling stressed. 'Enjoying loneliness' and 'friendship patterns' were two keywords obtained from the interviews with postgraduate students, showing the importance of a support network [30]. They were found to seldom look for the type of professional help 
offered by psychologists or counselors, which might have been made worse by language communication barriers [15].

Arguably, the category of leisure and entertainment could encompass many activities which might be carried out in green spaces. Indeed, as previously mentioned, the main activities undertaken in green space were related to the coping strategies students cite, such as relaxation, social activities, and physical activities. Among the responses, 'taking a walk' was reported to be the most popular activity taking part in green spaces, supporting the conclusion from previous literature that walking in nature might be the most important coping strategy conducted in urban green spaces [60].

Additionally, having picnics and engaging in other kinds of social activities can also be crucial in terms of stress reduction. Participating in activities held in green spaces might help Chinese students better integrate into the local culture and thus lower some of the transitional and adaptive stress experienced by this population.

\subsection{Limitations}

Generally, this was an exploratory study of an understudied population, and as such, its results should be seen as preliminary.

This study was first limited by its cross-sectional design, as it can only establish correlations between variables but cannot demonstrate causality. Although the study was inspired by previous experimental studies which have, under controlled conditions, collected an account of other coping strategies and reached similar findings, remaining confounding factors still exist. Participating in physical activities and other kinds of strategies, other environmental characteristics in the living environments, and students' lifestyles might also have an impact on their general health and perceived stress levels. It is also possible that the observed relationship between the use of green spaces and health might go the other way, with happy and relaxed people having more time and energy to visit green spaces.

Due to the exploratory nature of this study, the sample size is also limited. The recruitment was done by advertising in the WeChat group of Chinese students of 2018, most of whom were postgraduate students. Participants mainly consisted of students who had stayed in Edinburgh for less than a year. Owing to the lack of information about the gender distribution of the Chinese students present in Edinburgh, it is impossible to define whether the sample is representative of the population of Chinese students in 2018. Official data from the University of Edinburgh shows that in 2018/2019, 61\% of its student population was female, and $39 \%$ was male, which is in line with the gender distribution in our sample (65-35) [61]. Nevertheless, a truly representative sample might have found significant differences by gender in green space use and/or stress levels. In addition, the obtained response rate was $17 \%$ which was quite low and may have been due to a number of students leaving after the end of teaching.

Moreover, there are several limitations regarding the variable measurements. Indeed, accessibility was only measured as perceived and not objective. Similarly, the distance between homes and green spaces was measured through self-reports of the time needed to walk between the two. The measured variable was, therefore, the perceived travel time rather than the objective distance. The measure of accessible gardens around the homes was also self-reported and, as such, might not be completely accurate. Similarly, regarding the study of participants' preferred places, the survey required them to name the green spaces they frequently visited. This may have limited the reporting since some respondents might have only submitted the places for which they knew the names.

The measurement of the awareness of the benefits of green spaces for health and wellbeing through a single question was also limited. In this exploratory study on an understudied population, this variable was merely used to tease out the cultural element behind the motivation for the use of outdoor spaces. As it was initially hypothesized that Chinese students might not share this belief, we expected this variable to have provided an explanation for the participants' use or lack of use of green spaces. However, by simply 
asking the participants a yes/no question on whether they regarded visiting green spaces to be a way to relieve stress, the survey could not capture their actual beliefs regarding the benefits of green spaces. Phrasing the question as a closing statement might also have created a bias whereby students felt they had to answer in the positive. Further research could focus on delving deeper into this construct by conducting interviews with selected participants.

Overall, the use of self-reported data, although common in studies investigating green spaces and health $[3,5,11,12,19,22,56,57]$, does raise some questions on the validity of the data which was collected. Regarding University students, a population more likely to engage in risky behaviors such as hazardous alcohol use [28], it is possible that the activities participants shared in the survey was not truthful. This lack of truthfulness might be compounded by the cultural background of our participants, as social responsibilities bear more weight in collectivistic cultures [39]. However, since the survey was anonymous, we expect the social desirability bias to be moderate.

Additionally, the survey was carried out during the summer, and the data participants reported only related to the previous month. This was necessary for the health-related variables as the PSS only covers issues experienced during that time frame. As the weather was cited as a barrier by $12 \%$ of the participants who did not visit green spaces, it is expected that the results might be different during the winter.

\subsection{Future Work}

According to the existing findings and limitations in the study and the previous literature, an array of perspectives for future work is presented below.

Further studies could explore the potential aspects of green spaces influencing Chinese students' health and wellbeing when studying abroad. Using objective indicators of green spaces, such as locations of residential and green spaces acquired by satellite maps and carefully detailing the qualities of the environments, would help to assess the relationship between types of green spaces and students' responses. As Edinburgh is generally recognized for the quality of its green spaces [52], comparative studies considering other cities could also tease out the role of environmental characteristics in stress mitigation for this population.

It is also necessary for future studies to explore in more depth the potential influencing factors of the use of green space by Chinese students-for example, childhood experiences of contact with nature.

Similarly, the results obtained regarding the importance of the awareness of the benefits of green space for the health and wellbeing of students should be investigated further.

Finally, it would be relevant to carry out a comparative study between local or Western and international Chinese students to tease out the cultural differences between the two populations and understand in more detail the specificity of this vulnerable community.

\subsection{Implications for Practice}

On the basis of the obtained results, feasible strategies concerning the potential use of green spaces in Edinburgh or elsewhere in the UK for the health and wellbeing of international Chinese students could be proposed.

Based on the evidence supporting the health benefits of being in contact with local green spaces for Chinese students, relevant organizations at the university should conduct more outdoor events and courses, especially during the period of examinations, to help students cope with stress. Apart from this support, universities should also encourage Chinese international students to enjoy nature and local settings. Events in relation to physical activities and social interactions, such as ball games and BBQ, undertaken in the green spaces around campus could provide more opportunities for students to get more access to nature.

As there is not enough evidence pointing to the relationships between specific environmental characteristics, students' preferences, or any health benefits, the current findings 
are not enough to provide practical suggestions for landscape architects. Nevertheless, taking a walk and resting might be two crucial factors linking green spaces and health promotion, as was explored in previous work in Europe [55]. Designers, planners, and local policymakers could therefore pay particular attention to promoting these activities in local green spaces through their design. Consistent findings regarding the distance to green spaces being a barrier to use suggest that green spaces should be located as close as possible to the environment of the student's daily activities. Where it is not possible, views of the natural environment should be promoted. Similarly, on-street vegetation has been shown to improve health and wellbeing in dense urban contexts, so this solution could also be applied when space is limited [62].

Finally, the findings regarding the awareness of the health benefits of green spaces being a predictor of use could provide reliable evidence that university institutions and associations could help Chinese international students by communicating these benefits to students who might otherwise be unaware.

\section{Conclusions}

This study expands the existing evidence based on the links between urban green spaces and health to include international Chinese students living in the UK. This growing population is known to experience significant stress related to adaptative and transitional challenges but is currently understudied. This new data shows a strong association between urban green spaces and students' health status, supporting the existing corpus of evidence on the health benefits of natural environments. Frequency and length of visits to green space were found to be correlated with the students' perceived general health and perceived stress, regardless of gender, age, or length of stay in the host country. Furthermore, two significant influencing factors of the use of green spaces were confirmed in the study, including the perceived travel time to the nearest green space and a view of the green space from home. The qualitative survey also revealed that lifestyle, a lack of interest in visits, and lack of time were significant obstacles preventing students from visiting. Chinese international students were also found to be more likely to use leisure and entertainment activities such as listening to music and playing video games as a way to cope with stress. This study highlights the importance of raising awareness of the benefits of urban green spaces for stress management and wellbeing. Such findings provide some evidence-based suggestions for students, university organizations, and urban planners to address the health issues and stress levels faced by international Chinese students.

Author Contributions: Methodology, S.Z.; software, S.Z. and A.P.; investigation, S.Z.; writingoriginal draft preparation, S.Z.; writing-review and editing, S.Z. and A.P.; supervision, A.P. All authors have read and agreed to the published version of the manuscript.

Funding: This research received no external funding.

Institutional Review Board Statement: The study was conducted in accordance with the Declaration of Helsinki, and approved by the Ethics Committee of Edinburgh College of Art Postgraduate Office (Response ID 298668-298660-47800826, Completion date 9 June 2019).

Informed Consent Statement: Informed consent was obtained from all subjects involved in the study.

Data Availability Statement: The data presented in this study are available on request from the corresponding author. The data is not publicly available due to ethical restrictions.

Acknowledgments: The authors wish to thank Catharine Ward Thompson and Simon Bell for their supervision of the original study. We also wish to express our gratitude to the reviewers for their time and insightful comments.

Conflicts of Interest: The authors declare no conflict of interest. 


\section{Appendix A. Questionnaire}

Survey on the Use of Green Spaces by Chinese International Students and Their Levels of Stress

\section{INSTRUCTIONS:}

This questionnaire survey asks you about the ways you manage stress as a Chinese overseas student studying in Edinburgh, and the actual or potential use of the city's green spaces in helping you to cope. This questionnaire has been subject of a University of Edinburgh ethical review, and deemed appro-priate in the context of this project.

If you feel stressful or depressed, we advise you to contact your GP or any counseling services like Informatics Student Support. Contact details: Work: 0131 XXX 3148 (Lee K.)/0131 XXX 3151 (Da-vidson K.)/0131 XXX 2959 (Dornan I.) Email: inf-XXX@inf.ed.ac.uk. At the same time, if you have any questions about this study please do not hesitate to contact the researcher, Song Zhao, by email: s1809285@ed.ac.uk.

The information collected will be entirely anonymous and the data will only be used for the research-er's dissertation and no secondary use of any kind. You are free to leave the survey at any time if you feel upset or uncomfortable. If you are willing to participate in this study, please place an " $X$ " in the box below before starting to answer the questionnaire.

$\checkmark$ I agree to participate in this survey and I understand that I may withdraw at any time and that I will remain completely anonymous and that no personal information which could identify me will be collected.

\section{Part A Individual Information}

1. Your gender:

$\square$ Male $\square$ Female

2. Your age:

$\square$ Under 20 years old $\square 20-25$ years old $\square$ Over 25 years old

3. How long have you been in Edinburgh?

$\square$ Less than one year $\square 1$ to 2 years $\square$ More than 2 years

\section{Part B Health Status and Coping Strategies}

4. Please rate your own current general health level, 1 to 5 represents very unhealthy to very healthy.

\section{$\square 1 \square 2 \square 3 \square 4 \square 5$}

5. The following questions in this scale ask you about your feelings and thoughts during THE LAST MONTH. In each case, please indicate your response by placing an " $X$ " over the circle representing HOW OFTEN you felt or thought a certain way.

6. What ways do you usually cope with stress (for example talking to someone, listening to music, going for a walk etc)? Please write your answer in the order of the most used to the least used.

\section{Part C Use of Green Space}

Urban green space includes areas such as parks, sports fields as well as woods and natural meadows, wetlands or other ecosystems. For Edinburgh, the Meadows, Princes Street Garden and the George Square Garden as public parks, Calton Hill and Arthur's Seat as natural environments, or a private garden around your home, fall within the scope of urban green space.

7. Do you regard visiting green spaces to be a way to relieve stress?

$\square$ Yes $\square$ No

8. How often have you visited green space in Edinburgh in the last 4 weeks?

$\square$ Never $\square$ Once a week $\square 2$ to 3 times per week 
$\square 4$ to 5 times per week $\square$ More than 6 times per week

If you select "Never", please go to question $8 \mathrm{a}$ and then go to questions 11-14; If you select other options, please go to question $8 \mathrm{~b}$.

8a. What is the reason you don't visit a any green space in Edinburgh?

Please write your answer here

$8 \mathrm{~b}$. What do you like to do in green space?

Please write your answer in the order of most often to least often

9. How much time do you usually spend in a green space when you visit?

$\square$ Less than $30 \mathrm{~min} \square 30 \mathrm{~min}$ to an hour $\square 60$ to $90 \mathrm{~min}$

$\square 90$ to $120 \mathrm{~min} \square$ more than $120 \mathrm{~min}$

10. Please state the name of the green space you most frequently visit.

Please write your answer here

\section{Part D Access to Nature from Homes}

11. Please rate the quality of the urban green space you most frequently visit in Edinburgh, 1 represents very poor and 5 represents very good.

\section{$\square 1 \square 2 \square 3 \square 4 \square 5$}

12. How long does it take you to walk to the nearest green space from where you live?

$\square$ Less than $5 \mathrm{~min} \square 5$ to $10 \mathrm{~min}$

$\square 10$ to $15 \mathrm{~min} \square$ More than $15 \mathrm{~min}$

13. Do you have a view to green space or the distant countryside or sea from your home?

$\square$ Yes $\square$ No

14. Is there a garden around your home?

$\square$ Yes $\square$ No

1. In the last month, how often have you been upset because of something that happened unexpectedly?

2. In the last month, how often have you felt that you were unable to control the important things in your life?

3. In the last month, how often have you felt nervous and "stressed"?

4. In the last month, how often have you felt confident about your ability to handle your personal problems?

5. In the last month, how often have you felt that things were going your way?

6. In the last month, how often have you found that you could not cope with all the things that you had to do?

7. In the last month, how often have you been able to control irritations in your life?

8. In the last month, how often have you felt that you were on top of things?

9. In the last month, how often have you been angered because of things that were outside your control?

10. In the last month, how often have you felt difficulties were piling up so high that you could not overcome them?

\begin{tabular}{|c|c|c|c|c|}
\hline $\begin{array}{l}\text { Never } \\
0\end{array}$ & $\begin{array}{c}\text { Almost Never } \\
1\end{array}$ & $\begin{array}{c}\text { Sometimes } \\
2\end{array}$ & $\begin{array}{c}\text { Fairly Often } \\
3\end{array}$ & $\begin{array}{c}\text { Very Often } \\
4\end{array}$ \\
\hline 0 & 0 & 0 & 0 & 0 \\
\hline 0 & 0 & 0 & 0 & 0 \\
\hline 0 & 0 & 0 & 0 & $\bigcirc$ \\
\hline 0 & 0 & 0 & 0 & 0 \\
\hline 0 & 0 & 0 & 0 & 0 \\
\hline 0 & 0 & 0 & O & O \\
\hline 0 & O & 0 & 0 & $\bigcirc$ \\
\hline 0 & 0 & 0 & 0 & 0 \\
\hline 0 & 0 & 0 & 0 & 0 \\
\hline 0 & 0 & 0 & 0 & 0 \\
\hline
\end{tabular}




\section{References}

1. World Health Organization. Urban Green Spaces and Health; WHO Regional Office for Europe: Copenhagen, Denmark, 2016; Available online: https:/ / www.euro.who.int/_data/assets/pdf_file/0005/321971/Urban-green-spaces-and-health-reviewevidence.pdf (accessed on 6 April 2021).

2. Maas, J.; van Dillen, S.M.; Verheij, R.A.; Groenewegen, P.P. Social contacts as a possible mechanism behind the relation between green space and health. Health Place 2009, 15, 586-595. [CrossRef]

3. de Vries, S.; van Dillen, S.M.E.; Groenewegen, P.P.; Spreeuwenberg, P. Streetscape greenery and health: Stress, social cohesion and physical activity as mediators. Soc. Sci. Med. 2013, 945, 26-33. [CrossRef]

4. de Vries, S. Nearby nature and human health: Looking at mechanisms and their implications. In Innovative Approaches to Researching Landscape and Health; Thompson, C.W., Aspinall, P., Bell, S., Eds.; Routledge: London, UK, 2010 ; pp. 79-98.

5. Dadvand, P.; Bartoll, X.; Basagaña, X.; Dalmau-Bueno, A.; Martínez, D.; Ambros, A.; Cirach, M.; Triguero-Mas, M.; Gascon, M.; Borrell, C.; et al. Green spaces and General Health: Roles of mental health status, social support, and physical activity. Environ. Int. 2016, 91, 161-167. [CrossRef]

6. Qiu, L.; Nielsen, A.B. Are Perceived Sensory Dimensions a Reliable Tool for Urban Green Space Assessment and Planning? Landsc. Res. 2015, 40, 834-854. [CrossRef]

7. Chen, H.; Qiu, L.; Gao, T. Application of the eight perceived sensory dimensions as a tool for urban green space assessment and planning in China. Urban For. Urban Green. 2019, 40, 224-235. [CrossRef]

8. Roy, S.; Byrne, J.; Pickering, C. A systematic quantitative review of urban tree benefits, costs, and assessment methods across cities in different climatic zones. Urban For. Urban Green. 2012, 11, 351-363. [CrossRef]

9. Ward Thompson, C.; Aspinall, P. Natural environments and their impact on activity, health, and quality of life. Appl. Psychol. Health Well-Being 2011, 3, 230-260. [CrossRef]

10. Ward Thompson, C.; Roe, J.; Aspinall, P.; Zuin, A.; Travlou, P.; Bell, S. Community Green: Using Local Spaces to Tackle Inequality and Improve Health; Commission for Architecture and the Built Environment (CABE): London, UK, 2010.

11. Ward Thompson, C.; Roe, J.; Aspinall, P.; Mitchell, R.; Clow, A.; Miller, D. More green space is linked to less stress in deprived communities: Evidence from salivary cortisol patterns. Landsc. Urban Plan 2012, 105, 221-229. [CrossRef]

12. Ward Thompson, C.; Aspinall, P.; Roe, J.; Robertson, L.; Miller, D. Mitigating stress and supporting health in deprived urban communities: The importance of green space and the social environment. Int. J. Environ. Res. Public Health 2016, 13, 440. [CrossRef] [PubMed]

13. Roe, J.; Aspinall, P.; Thompson, C.W. Coping with Stress in Deprived Urban Neighborhoods: What Is the Role of Green Space According to Life Stage? Front. Psychol. 2017, 8, 1760. [CrossRef]

14. Sherry, M.; Thomas, P.; Chui, W.H. International students: A vulnerable student population. High. Educ. 2010, 60, 33-46. [CrossRef]

15. Wei, M.; Heppner, P.P.; Mallen, M.J.; Ku, T.Y.; Liao, K.Y.H.; Wu, T.F. Acculturative Stress, Perfectionism, Years in the United States, and Depression Among Chinese International Students. J. Couns. Psychol. 2007, 54, 385-394. [CrossRef]

16. Ulrich, R. View through a window may influence recovery from surgery. Science 1984, 224, 420-421. [CrossRef]

17. Ulrich, R.; Simons, R.F.; Losito, B.D.; Fiorito, E.; Miles, M.A.; Zelson, M. Stress recovery during exposure to natural and urban environments. J. Environ. Psychol. 1991, 11, 201-230. [CrossRef]

18. Korpela, K.M. Negative Mood and Adult Place Preference. Environ. Behav. 2003, 35, 331-346. [CrossRef]

19. Grahn, P.; Stigsdotter, U.A. Landscape planning and stress. Urban For. Urban Green. 2003, 2, 1-18. [CrossRef]

20. Hartig, T.; Mitchell, R.; de Vries, S.; Frumkin, H. Nature and Health. Annu. Rev. Public Health 2014, 35, 207-228. [CrossRef] [PubMed]

21. Li, D.; Sullivan, W.C. Impact of views to school landscapes on recovery from stress and mental fatigue. Landsc. Urban Plan. 2016, 148, 149-158. [CrossRef]

22. Nielsen, T.S.; Hansen, K.B. Do green areas affect health? Results from a Danish survey on the use of green areas and health indicators. Health Place 2007, 13, 839-850. [CrossRef]

23. Ward Thompson, C.; Aspinall, P.; Montarzino, A. The Childhood Factor: Adult visits to green places and the significance of childhood experience. Environ. Behav. 2008, 40, 111-143. [CrossRef]

24. Hadavi, S. Direct and Indirect Effects of the Physical Aspects of the Environment on Mental Well-Being. Environ. Behav. 2017, 49, 1071-1104. [CrossRef]

25. Cohen, S.; Kamarck, T.; Mermelstein, R. A Global Measure of Perceived Stress. J. Health Soc. Behav. 1983, 24, 385-396. [CrossRef] [PubMed]

26. Hamaideh, S.H. Stressors and Reactions to Stressors Among University Students. Int. J. Soc. Psychiatry 2011, 57, 69-80. [CrossRef]

27. Wang, X.; Rodiek, S.; Wu, C.; Chen, Y.; Li, Y. Stress recovery and restorative effects of viewing different urban park scenes in Shanghai, China. Urban For. Urban Green. 2016, 15, 112-122. [CrossRef]

28. Andersson, C.; Johnsson, K.O.; Berglund, M.; Öjehagen, A. Stress and hazardous alcohol use: Associations with early dropout from university. Scand. J. Public Health 2009, 37, 713-719. [CrossRef] [PubMed]

29. Pereira, S.; Reay, K.; Bottell, J.; Walker, L.; Dzikit, C.; Platt, C.; Goodrham, C. University Student Mental Health Survey 2018; The Insight Network and Dig-In: London, UK, 2019. 
30. Gu, Q. Maturity and Interculturality: Chinese students' experiences in UK higher education. Eur. J. Educ. 2009, 44, 37-52. [CrossRef]

31. Yuan, F.Y. A Study on the Psychological Causes of Intercultural Stress among International Students. Jianghuai Forum 2010, 6 , 151-155.

32. Spencer-Oatey, H.; Xiong, Z. Chinese Students' Psychological and Sociocultural Adjustments to Britain: An Empirical Study. Lang. Cult. Curric. 2006, 19, 37-53. [CrossRef]

33. Higher Education Student Statistics. UK, 2019/20-Where Students Come From and Go To Study. 2021. Available online: https:/ / www.hesa.ac.uk/news/27-01-2021/sb258-higher-education-student-statistics/location (accessed on 8 April 2021).

34. Gentin, S. Outdoor recreation and ethnicity in Europe-A review. Urban For. Urban Green. 2011, 10, 153-161. [CrossRef]

35. Holt, E.; Lombard, Q.; Best, N.; Smiley-Smith, N.; Quinn, J. Active and Passive Use of Green Space, Health, and Well-Being amongst University Students. Int. J. Environ. Res. Public Health 2019, 16, 424. [CrossRef]

36. Walker, G.J.; Deng, J.; Dieser, R.B. Ethnicity, acculturation, self-construal, and motivations for outdoor recreation. Leis. Sci. 2001, 23, 263-283. [CrossRef]

37. Martin, L.; White, M.P.; Hunt, A.; Richardson, M.; Pahl, S.; Burt, J. Nature contact, nature connectedness and associations with health, wellbeing and pro-environmental behaviours. J. Environ. Psychol. 2020, 68, 101389. [CrossRef]

38. Zhang, W.; Goodale, E.; Chen, J. How contact with nature affects children's biophilia, biophobia and conservation attitude in China. Biol. Conserv. 2014, 177, 109-116. [CrossRef]

39. Krettenauer, T.; Wang, W.; Jia, F.; Yao, Y. Connectedness with nature and the decline of pro-environmental behavior in adolescence: A comparison of Canada and China. J. Environ. Psychol. 2020, 71, 101348. [CrossRef]

40. Berto, R. Assessing the restorative value of the environment: A study on the elderly in comparison with young adults and adolescents. Int. J. Psychol. 2007, 42, 331-341. [CrossRef]

41. Patuano, A. Biophobia and Urban Restorativeness. Sustainability 2020, 12, 4312. [CrossRef]

42. Yang, T.; Barnett, R.; Fan, Y.; Li, L. The effect of urban green space on uncertainty stress and life stress: A nationwide study of university students in China. Health Place 2019, 59, 102199. [CrossRef]

43. Xi, W.; Yanlong, Z.; Zhao, R.; Niu, L. Study on the Effects of Four Campus Green Landscapes on College Students' Physiological and Psychological Indicators. Chin. Landsc. Archit. 2020, 36, 92-97.

44. Liu, Q.; Zhang, Y.; Lin, Y.; You, D.; Zhang, W.; Huang, Q.; van den Bosch, C.C.K.; Lan, S. The relationship between self-rated naturalness of university green space and students' restoration and health. Urban For. Urban Green. 2008, 34, 259-268. [CrossRef]

45. Chen, L.; Peng, C.; Luo, B. Application of Environmental Psychology in Landscape Design of University Campus. J. Green Sci. Technol. 2017, 15, 7-9.

46. Jiangping, W.; Zhou, Z. Study on Campus Landscape Design from the Founction of Stress Relief. Huazhong Arch. 2011, 2, 101-103.

47. Speake, J.; Edmondson, S.; Nawaz, H. Everyday encounters with nature: Students' perceptions and use of university campus green spaces. Hum. Geogr. J. Stud. Res. Hum. Geogr. 2013, 7, 21-31. [CrossRef]

48. Mcfarland, A.L.; Waliczek, T.M.; Zajicek, J.M. The Relationship Between Student Use of Campus Green Spaces and Perceptions of Quality of Life. Am. Soc. Hortic. Sci. 2008, 18, 232-238. [CrossRef]

49. Lottrup, L.; Grahn, P.; Stigsdotter, U.K. Workplace greenery and perceived level of stress: Benefits of access to a green outdoor environment at the workplace. Landsc. Urban Plan. 2013, 110, 5-11. [CrossRef]

50. Laatikainen, T.; Tenkanen, H.; Kyttä, M.; Toivonen, T. Comparing conventional and PPGIS approaches in measuring equality of access to urban aquatic environments. Landsc. Urban Plan. 2015, 144, 22-33. [CrossRef]

51. Höglhammer, A.; Muhar, A.; Stokowski, P. Access to and use of the Wienerwald Biosphere Reserve by Turkish and Chinese people living in Austria-implications for planning. J. Prot. Mt. Areas Res. Manag. 2019, 11, 11-17. [CrossRef]

52. BBC News: Edinburgh Is Top UK City for Green Space. Available online: https://www.bbc.co.uk/news/uk-scotland-38522414 (accessed on 13 July 2019).

53. Ordnance Survey: OS Maps: Online Mapping and Walking, Running and Cycling Routes. Available online: https://osmaps ordnancesurvey.co.uk/55.93503,-3.16636,12 (accessed on 20 July 2019).

54. The University of Edinburgh, 2019. Student Numbers 2017/18. Available online: https://www.ed.ac.uk/about/annual-review/ student-numbers (accessed on 13 July 2019).

55. Ware, J.; Sherbourne, C.D. The MOS 36-Item Short-Form Health Survey (SF-36): I. Conceptual Framework and Item Selection. Med. Care 1992, 30, 473-483. [CrossRef] [PubMed]

56. Maas, J. Green space, urbanity, and health: How strong is the relation? J. Epidemiol. Community Heal. 2006, 60, 587-592. [CrossRef]

57. Maas, J.; van den Berg, A.; Verheij, R.A.; Groenewegen, P.P. Green space as a buffer between stressful life events and health. Soc. Sci. Med. 2010, 70, 1203-1210.

58. Guest, G.; MacQueen, K.; Namey, E. Applied Thematic Analysis; SAGE Publications, Inc.: Thousand Oaks, CA, USA, 2012.

59. Barton, J.; Pretty, J. What is the Best Dose of Nature and Green Exercise for Improving Mental Health? A Multi-Study Analysis. Environ. Sci. Technol. 2010, 44, 3947-3955. [CrossRef]

60. Stigsdotter, U.K.; Grahn, P. Stressed individuals' preferences for activities and environmental characteristics in green spaces. Urban For. Urban Green. 2011, 10, 295-304. [CrossRef] 
61. The University of Edinburgh. Factsheet 2018/2019. 2019. Available online: http://www.docs.sasg.ed.ac.uk/gasp/factsheet/ Student_Factsheet_31072019.pdf (accessed on 26 November 2021).

62. Tiwary, A.; Williams, I.D.; Heidrich, O.; Namdeo, A.; Bandaru, V.; Calfapietra, C. Development of multi-functional streetscape green infrastructure using a performance index approach. Environ. Pollut. 2016, 208, 209-220. [CrossRef] [PubMed] 